\title{
Bone Metastases: An Overview
}

\author{
Filipa Macedo ${ }^{*}$, Katia Ladeira ${ }^{2}$, Filipa Pinho ${ }^{2}$, Nadine Saraiva ${ }^{1}$, Nuno Bonito ${ }^{1}$, Luísa Pinto ${ }^{2}$ and Francisco \\ Gonçalves $^{2}$
}

${ }^{1}$ Medical Oncology Department, Portuguese Oncology Institute of Coimbra, Portugal

${ }^{2}$ Internal Medicine Department, Braga Hospital, Portugal

Submission: March 28, 2017; Published: April 10, 2017

"Correspondence Address: Filipa Macedo, Medical Oncology Department, Portuguese Oncology Institute of Coimbra, Avenida Bissaya Barreto Coimbra, Portugal, Tel: +351 916842232; Email: filipa.c.macedo@gmail.com

\begin{abstract}
Bone is a frequent site of metastases and typically indicates a short--term prognosis in cancer patients. Once cancer has spread to the bones it can rarely be cured, but often it can still be treated to slow its growth. The majority of skeletal metastases are due to breast and prostate cancer. Bone metastasis is actually much more common than primary bone cancers, especially in adults. The diagnosis is based on signs, symptoms and imaging. New classes of drugs and new interventions are given a better quality of life to these patients and improved the expectancy of life. It is necessary a multidisciplinary approach to treat patients with bone metastasis. In this paper we review the types, clinical approach and treatment of bone metastases.
\end{abstract}

Keywords: Bone metastasis; Fractures; Bisphosphonates; Denosumab; Radiotherapy

\section{Introduction}

Metastasize is a process that involves loss of intercellular cohesion, cell migration, angiogenesis, access to systemic circulation, survival in circulation, evasion of local immune responses, and growth at distant organs [1,2]. Bone is the third most frequent site of metastasis, behind lung and liver [3]. Breast and prostate cancer are responsible for the majority of the skeletal metastases (up to 70\%) [4]. This reflects both the high incidence and relatively long clinical course of these tumors.

The overall incidence of bone metastasis is not known [3]. The relative incidence of bone metastasis by type of tumor, in patients with advanced metastatic disease, is: $65--75 \%$ in breast; $65--75 \%$ in prostate; $60 \%$ in thyroid; $30--40 \%$ in lung; $40 \%$ in bladder; $20--25 \%$ in renal cell carcinoma and $14--45 \%$ in melanoma. The median survival from diagnosis of bone metastasis is: 6 months in melanoma; 6--7 months in lung; 6--9 months in bladder; 12 months in renal cells carcinoma; $12--53$ months in prostate; $19--25$ months in breast and 48 months in thyroid [5]. Bone metastases are a major cause for morbidity, characterized by severe pain, impaired mobility, pathologic fractures, spinal cord compression, bone marrow aplasia and hypocalcaemia [4].

\section{Types of Bone Metastasis}

Bone metastasis are classified as osteolytic, osteoblastic or mixed, according to the primary mechanism of interference with normal bone remodeling:
A. Osteolytic, characterized by destruction of normal bone, present in multiple myeloma, renal cell carcinoma, melanoma, non--small cell lung cancer, non--hodgkin lymphoma, thyroid cancer or langerhans cell histiocytosis. The great majority of breast cancers produce osteolytic metastases. This bone destruction is primarily mediated by osteoclasts and not a direct effect of tumor cells $[5,6]$. Other way, with lesser importance, is the compression of vasculature and consequent ischaemia in the late stages of cancer [3].

Parathyroid hormone - related peptide (PTHrP) has a major role in the development of osteolytic lesions [7]. It is unclear if bone microenvironment induces cancer cells to express PTHrP or if cells that metastasize to bone have an intrinsic higher PTHrP expression [8]. RANKL (receptor activator of NF-- kappaB ligand) play a critical role in the formation of osteoclasts by stimulating precursor cells when binds to RANK (receptor activator of NF-kappaB) receptor on the cell membrane of osteoclast precursors [9]. Osteoprotegerin (OPG) suppresses osteolysis by inhibition of RANKL binding to the RANK receptor. So, the ratio of RANKL to OPG regulates osteoclasts activity [5]. Once osteoclasts attach to the bone surface, they release proteases that resorb bone matrix.

B. Osteoblastic (or sclerotic), characterized by deposition of new bone, present in prostate cancer, carcinoid, small cell lung cancer, Hodgkin lymphoma, medulloblastoma or POEMS (polyneuropathy, organomegaly, endocrinopathy, monoclonal 


\section{Cancer Therapy \& Oncology International Journal}

gammopathy and skin changes) syndrome. The mechanisms of osteoblastic metastases are still poorly understood. In some instances the new bone formation is not necessarily preceded by bone resorption [3]. Transforming growth factor (TGF) B1, TGF-B2, bone morphogenic proteins (BMP) and endothelin--1 are associated with osteoblast generation [10]. Prostate--specific antigen (PSA), can cleave PTHrP, allowing the osteoblastic reaction predominate by decreasing bone reabsorption [11]. Core binding factor alpha 1 (Cbfa1), also known as Runx--2, is linked to osteoblast differentiation [12].

C. Mixed, if a patient has both osteolytic and osteoblastic lesions, or if a individual metastasis has both osteolytic and osteoblastic components, present in breast cancer, gastrointestinal cancers and squamous cancers. Although breast cancer gives origin predominantly to osteolytic lesions, 15--20\% of women have osteoblastic lesions, or both type of lesions [13].

\section{Mechanisms of Metastases to Bone}

Bone metastasis are almost always multiple and involve axial skeleton4. It has been suggested that this distribution might be in relation to the hematopoietically active red bone marrow [13]. There exists a para-- vertebral network that may play a role in the development of bone metastasis [14]. This theory is supported by the high incidence of bone metastases without corresponding lesions in the lung (suggest an alternative pathway of spread). In addiction, the microenvironment must be favorable for tumor cell survival [4].

Once the tumor cell is in circulation, it needs:

\section{Vascular adhesion and extravasation}

The cell interacts with endothelium in order to extravasate and stay in a specific tissue [15]. Chemo attractive and adhesion molecules play a fundamental role in this selective retention of cancer cells in bone marrow vasculature. Cancer cells use equivalent molecules to vascular cell adhesion molecules (VCAM) and E--selectin to adhere to endothelium [16,17]. We also know that chemokines (chemoattractive cytokines), integrins, osteopontin, bone sialoprotein and type I collagen are critical for organ colonization by cancer cells $[18,19]$. Examples of such interactions are: expression of CXCR4 (chemokine receptor) by neuroblastoma tumors that mediates the attachment to SDF-1 in bone (stromal--cell derived factor 1 or CXCL12) [20]; expression of RANK by breast adenocarcinoma that mediates the attachment to RANKL in bone [21]; expression of sialoprotein by non--small cell lung cancer that facilitate binding to collagen type I in bone [22].

\section{Micro--environmental support}

The "seed and soil" hypothesis tells us that the microenvironment provides a fertile ground ("the soil"), for the survival and growth of metastatic cancer cells ("the seed") [23]. Bone specific microenvironment is constituted by osteoclasts, osteocytes, osteoclasts and stroma. The bone formation and reabsorption release and activate survival and growth promoting factors that may contribute to bone metastases development [24].

\section{Epithelial - Mesenchymal transition}

Normal cell can lose their epithelial features and acquire mesenchymal characteristics. This process is called Epithelial-Mesenchymal Transition, and enables epithelial cells to migrate to a new environment. This occurs mainly during embryogenesis, but in cancer cells this process confers the invasive phenotype [4].

\section{Clinical Aspects}

The median survival of patients after first bone metastasis by breast cancer is 20 months. This is in marked contrast to those with metastases of breast cancer in the liver, 3 months, or with bone metastases from non-- small cell lung cancer, 6 months [25]. Patients with bone only disease are more likely at diagnosis to be older, post--menopausal woman with lobular carcinoma, with little or no involvement of axillary lymph nodes. Factors that predict for a longer survival are estrogen receptor positivity, grade I and II ductal or lobular cancers (instead of grade III), a long disease--free interval ( $>3$ years) and a pre-menopausal status [26].

In prostate cancer, men with a good performance status and bone only disease, affecting predominantly the axial skeleton, have a median duration of disease control after androgen blockade of 4 years and a median survival of 53 months. This is in marked contrast to those with visceral disease with a median survival of 30 months and 12 months with visceral disease and poor performance status [27].

Bone metastases are a major cause for morbidity, characterized by severe pain, impaired mobility, pathologic fractures, spinal cord compression, bone marrow aplasia and hypocalcaemia [4].

Hypercalcemia is the most common metabolic complication of malignant disease28. It is frequent in squamous call carcinomas of the lung, adenocarcinoma of the breast and kidney, multiple myeloma and lymphoma. The four main contributes to hypocalcaemia are: focal osteolysis by tumor cells, generalized osteolysis by humoral factors secreted by the tumor, increased renal tubular reabsorption of calcium and impaired renal glomerular function. Breast cancer secretes PTHrP; multiple myeloma leads to impaired renal function due to deposition of Bence--Jones proteins; some lymphomas produce active metabolites of vitamin $\mathrm{D}$, which increases both bone resorption and intestinal absorption of calcium [28].

Moderate to severe hypocalcaemia, if left untreated, causes a number of unpleasant symptoms related to dysfunction of the gastrointestinal tract, kidneys and central nervous systems such as constipation, polyuria, polydipsia and fatigue. In final stages, hypocalcaemia can leads to cardiac arrhythmias and acute renal 


\section{Cancer Therapy \& Oncology International Journal}

failure [3]. With hypercalcemia, parathyroid hormone levels are suppressed, and PTHrP may be elevated. This leads to increased osteoblastic bone resorption. Hypercalcaemia carries a poor prognosis with a median survival of 10--12 weeks [5].

Pathologic fractures occur in 10--30\% of all cancer patients, with proximal parts of the long bones being the most frequent fracture site, and the femur accounting for over half of all cases5. Rib fractures and vertebral collapses are also very common, which can lead to kyphoscoliosis and a degree of restrictive lung disease [29]. Breast cancer accounts for $60 \%$ of the pathologic fractures and lung cancer for only $10 \%$ of cases [30]. The risk factors that increase fracture risk in post menopausal woman with breast cancer are: aromatase inhibitor treatment, bone mineral density (BMD) T--score $<--2,5,>65$ years, oral corticosteroid use for more than 6 months, low body mass index $\left(<20 \mathrm{~kg} / \mathrm{m}^{2}\right)$, family history of hip fracture, personal history of fragility fracture after age 50 and smoking [31].

The most disability is caused by a long bone fracture or epidural extension of tumor into the spine3.The probability of developing a pathological fracture increases with the duration of metastatic involvement. Although the intensity of bone pain is not directly associated with fracture risk, pain that is exacerbated by movement does appear to be an important factor in predicting impending fracture. Primary internal stabilization followed by radiotherapy is usually the treatment of choice [3].

Bone pain is the most common type of pain from cancer, is poorly localized, worse at night, not necessarily relieve with sleep or lying down [32]. In cancer patients, development of bone pain usually is considered to be highly suggestive of bone metastases [5]. The pain associated with bone metastasis could be either of inflammatory or mechanical origin. Inflammatory pain is related to the local release of cytokines and chemical mediators by the tumor cells, periosteal irritation, stimulation of intraosseous nerves. Mechanical pain is related to the pressure or mass effect of the tumor tissue within the bone, with loss of bone strength thus turning into activity--related pain [5]. The inhibition of osteoblastic bone reabsorption reduces bone pain [32]. The use of osteoclast inhibitors, such bisphosphonates and denosumab, reduce bone pain.

The development of back pain in a patient with cancer, associated with an abnormal spinal radiograph should warn the physician for possible spinal cord compression. It is more commonly seen in breast cancer (20-- 30\%) and lung cancer (15\%) [5]. For a successful rehabilitation the diagnosis must be fast, high--dose corticosteroids treatment, rapid assessment and urgent referral for both decompression and spinal stabilization. If compression is not relieved within 24--48 hours, neurologic recovery is unlikely $[3,28]$.

A basic screening must be performed when one of the signs and symptoms described above are present: a complete blood cell count to evaluate for anemia and myelosuppression; serum calcium, phosphorus, 25-- hydroxyvitamin D, alkaline phosphatase, creatinine, thyroid--stimulating hormone, protein electrophoresis and parathyroid hormone level to identify bone turnover and evaluate hypercalcemia [5,33]. This study must be complemented with imaging data [scintigraphic bone scans, plain x--rays, computed tomography (CT) scans or magnetic resonance imaging (MRI)] [5].

\section{Bone scintigraphy}

Is highly sensitive but usually has a low specificity. Sensitivity of $99 \mathrm{Tc}$ scintigraphy has been reported to range from 62 to $89 \%$, with a false--positive rate as high as $40 \%$. It is more sensitive and more specific than plain films and CT, while MRI is superior in evaluating vertebral metastases [34]. It provides information on osteoblastic activity and skeletal vascularity, with preferential uptake of tracer at sites of active bone formation that reflects the metabolic reaction of bone to the disease process, whether neoplastic, traumatic or inflammatory [33].

\section{Radiographs}

Are fast, cheap, and readily available techniques for evaluating bone metastases. Plain radiography should be the first test in the evaluation of bone pain. A plain radiography is very specific but sensitivity is low (44--50\%) because metastatic lesions may not appear on X--ray at initial stages. Lesions up to $1 \mathrm{~cm}$ might go undetected, while more than $50 \%$ of trabecular bone must be destroyed before it will be evident on film. Medullary lesions are more difficult to detect than lesions in cortical bone because of the limited contrast in trabecular bone [35]. Osteolytic lesions appear as a darker hole in the gray-white bone image; osteoblastic lesions appear as spots that are whiter than the bone around them.

The sensitivity of CT for the diagnosis of bone metastases ranges from 71 to $100 \%$ [36]. CT produces images with excellent soft tissue and contrast resolution. Bone destruction and sclerotic deposits are usually clearly shown and any soft tissue extension of bone metastases is easily visualized. CT is particularly useful to localize lesions for biopsy [33].

\section{MRI}

Is required to diagnose spinal cord compression and is useful in imaging bone marrow to assess involvement by the tumor. The sensitivity ranges from 82 to $100 \%$ and its specificity ranges from 73 to $100 \%$ [37].

\section{Positron emission tomography}

(PET) detects the presence of tumor directly by quantifying metabolic activity. It is superior to bone scintigraphy in the detection of bone metastases from lung cancer (sensitivity $92 \%$ and specificity 99\%) [38], and from breast cancer (sensitivity $95 \%$ and specificity 94\%) [39]. It has lower accuracy in renal and prostate cancer bone metastasis because they are slow growing (so, the uptake of 18-- fluorodeoxyglucose is low) 
[40]. PET permits earlier diagnosis of bone metastases in multiple myeloma, showing bone resorption sites undetected with conventional diagnostic methods. In addition, it can reveal metastatic spread to sites other than bone [41].

In at--risk patients, an assessment of clinical risk factors and screening for osteoporosis with the measurement of BMD by dual x--ray absorptiometry (DXA) is required. Osteoporosis is a systemic skeletal disease in both men and women characterized by low bone mass and micro architectural deterioration of bone tissue that results in a high risk of fracture. Estrogen deficiency is the major cause of accelerated bone loss leading to an increased incidence of fractures. Consequently estrogen deprivation in women with breast cancer and in men receiving androgen deprivation therapy will accelerate bone turnover leading to a decrease in BMD and a 40-- 50\% increase in fracture incidence. Treatment with anti--resorptives is recommended in all patients receiving aromatase inhibitor therapy with a T--score $<--2,0$ [33].

\section{Treatment}

Treatment decisions depend on several parameters, for example, if bone disease is localized or widespread, if there is evidence of extra skeletal metastases, the kind of cancer and his features (like estrogen receptors in breast cancer), prior treatment history and disease response, the symptoms and the general state of health [28]. Treatments can often shrink or slow the growth of bone metastases and can help with the symptoms they are causing but, they are not curative.

\section{Bisphosphonates}

Are analogues of pyrophosphate, a natural inhibitor of bone demineralization [28]. Bisphosphonates bind avidly to exposed bone mineral around resorbing osteoclast and this leads to very high local concentrations of product in the resoption lacunae. Then, bisphosphonates are internalized by the osteoclast causing disruption of the chemical process involved in bone resorption $[5,42]$. Bisphosphonates also cause osteoclast apoptosis and some studies suggest that they may also have direct apoptotic effects on tumour cells $[5,43]$. In oncology, bisphosphonates are the standard treatment for tumour--induced hypercalcaemia and a new form of therapy for bone metastasis [44].

With intravenous bisphosphonates and rehydration, 70$-90 \%$ of patients will achieve normocalcaemia. The effect on pain in bone metastases is independent of the nature of the underlying tumour and the sclerotic lesions respond similarly to lytic metastases [45]. The studies are mainly done in breast cancer and multiple myeloma; lung, kidney and prostatic cancer have few studies.

They are well tolerated. The most common adverse events include flu--like symptoms (fever, arthralgias, myalgias and weakness), anemia, nausea, bone pain, dyspnea and peripheral edema. These events are mostly limited and mild to moderate [5]. A rare but very serious side effect is osteonecrosis of the jaw.
All bisphosphonates undergo renal clearance so, patients with renal impairment (serum creatinine level $>3.0 \mathrm{mg} / \mathrm{dL}$ ) should not receive the treatment [46].

There are three generations of bisphosphonates: 1st generation, etidronate, clodronate, tiludronate; 2nd generation, pamidronate, alendronate, ibandronate; 3rd generation, risedronate, zoledronic acid. The approved ones are: oral clodronate at a daily dose of $1600 \mathrm{mg}$ and oral ibandronate $50 \mathrm{mg}$; intravenous (IV) pamidronate $90 \mathrm{mg}$ (infusion of $2 \mathrm{~h}$ ), ibandronate $6 \mathrm{mg}$ (infusion of $1 \mathrm{~h}$ ), zoledronic acid $4 \mathrm{mg}$ (infusion of $15 \mathrm{~min})$. Zoledronic acid is the newer bisphosphonate approved for multiple myeloma, lung, breast and prostate cancer with bone metastasis. It is 100--times more effective than pamidronate [47]. Patients doing these treatments should take a supplement containing calcium and vitamin D.

The two most studied bisphosphonates in oncology are clodronate (oral) and pamidronate (intravenous). Only <5\% of an oral dose of clodronate is absorbed, require a $1 \mathrm{~h}$ fasting period and is associated with gastrointestinal adverse events like diarrhea. It is less effective than IV pamidronate at reducing skeletal-- related events. Infusions of pamidronate are time-consuming and demand a therapy unit $[5,28]$.

\section{Denosumab}

Is a human monoclonal antibody that inhibits the RANKL, preventing the development of osteoclasts. It can help prevent or delay problems like fractures in patients with bone metastases at least as well as zoledronate, and is safe to give to patients with impaired renal function. It also can be helpful when zoledronate is no longer working. Side effects are similar to bisphosphonates, including nausea, diarrhea, weakness and can cause osteonecrosis of the jaw too [48].

Osteonecrosis of the jaw is more common when IV bisphosphonates or denosumab are administered on a monthly basis for control of metastases and is much less frequent with less intensive use of bisphosphonates or denosumab for preservation of bone mass. Osteonecrosis of the jaw management is mostly conservative, and healing occurred in more than one--third of patients. Most of the patients with confirmed osteonecrosis of the jaw had a history of tooth extraction (62\%), poor oral hygiene and/or use of a dental appliance [49].

Radiotherapy is the treatment of choice for localized bone pain, but in presence of poorly localized bone pain or recurrence of pain in previously irradiated skeletal sites, the bisphosphonates are an alternative treatment approach [28].

\section{External radiotherapy}

Provides excellent palliation for localized metastatic bone pain however, [50] the mechanism of pain relief after radiation therapy is poorly understood [28]. Pain relief usually occurs rapidly, with more than $50 \%$ of responders showing benefit within 1--2 weeks. If improvement in pain has not occurred by 


\section{Cancer Therapy \& Oncology International Journal}

6 weeks or more after treatment, it is unlikely to be achieved33. Indications for radiotherapy for bone metastases include pain, risk for pathologic fracture and neurological complications arising from spinal cord compression [5].

Radiation therapy can be delivered using three forms of treatment: local--field radiation therapy, wide--field radiation therapy and radionuclide therapy [28]. The local--field radiation therapy is considered the conventional treatment of bone metastases. It treats the involved bone and yields a pain relief rate of $80--90 \%$ [51]. Several randomized trials have indicated that a single fraction of 8Gy is adequate for pain relief.50 Wide field (half-- body, hemibody) radiation therapy can be used as primary palliative therapy for widespread symptomatic bone metastases or as an adjuvant to local--field radiation to reduce the later expression of occult metastases and to reduce the frequency of re--treatment $[52,53]$. It is possible to distinguish: upper wide--field treatments (from skull or C1 to L2--3) - optimal single--dose is 6Gy; mid--body wide--field treatments (from L1 to upper third of the femurs) - optimal single--dose is 8Gy; lower wide--field treatments (from L3--4 to above the knees)- optimal single--dose is 8Gy [51]. Wide--field radiation provides pain relief for $64--100 \%$ of patients and approximately $50--66 \%$ of patients maintain pain relief for the remainder of their lives. The radiation fields must be shaped to reduce exposure of sensitive structures such as lung, gut, kidney and liver.

\section{Radionuclide therapy}

Is the systemic use of radioisotopes for bone pain [28]. Radiopharmaceuticals like strontium--89, rhenium--186 or samarium--153, have been shown to be effective in palliation of metastatic bone pain. They are preferentially taken up at sites for bone formation, so they probably are most effective for osteoblastic metastases [54]. The principal side effects are myelosuppression and pain flare.

Recently we have the radium--223, calcium mimetic and alpha emitter that selectively binds to areas of increased bone turnover in bone metastases. It bounds into newly formed bone stroma and the radiation induces mainly double--stranded DNA breaks that result in a potent and highly localized cytotoxic effect. Toxic effects on adjacent tissues and particularly the bone marrow are minimal due to the short path of the alpha particles. Radium--223 significantly prolonged overall survival in patients who had castration--resistant prostate cancer and bone metastases, with a $30 \%$ reduction in the risk of death [55].

\section{Ablation}

Is the procedure where a needle or probe is introduced into a tumor and using heat, cold or a chemical, the tumor is destroyed. It may be used if only 1 or 2 bone tumors are causing symptoms. The most common types of ablation are radiofrequency ablation (RFA), where an electric current delivered through the needle heats the tumor to destroy it; and cryoablation, where a very cold probe is put into the tumor to freeze the cancer cells [56].
Although effective at reducing a patient's pain, RFA has a critical limitation, which is the nonvisualization of the ablation margin with CT monitoring. In contrast to RFA, the ablation zone of cryoablation is readily identified with CT imaging as a low-attenuation ice ball beyond which tissues are safe from thermal injury [57]. Another advantage of cryoablation relative to RFA is that patients treated with cryoablation do not experience increased pain during the procedure or in the immediate post treatment period [58].

Although the complication rate using RFA and cryoablation for treatment of painful metastases is low, it was reported neurologic injuries, neuropathic pain and infection in the treatment area [59]. For systemic antitumor treatment selection, the pathological type of the tumor is most important. In lymphoma and germ cell tumors involving bone chemotherapy can be curative, while in renal cell carcinoma or melanoma it has little effect [3].

Recently, there are Cabozantinib (XL184), an oral tyrosine kinase inhibitor whose targets include VEGFR2, MET, KIT, and mutationally activated RET. It was associated with high rates of bone scan improvement, but the side effects required dose reduction or treatment discontinuation. In addition, statistically significant improvement in progression--free survival was seen with cabozantinib compared with placebo, in metastatic prostate cancer [60].

\section{Surgery}

Is only indicated for fractures of long bones and hip joints, in spinal cord involvement, or peripheral nerve compression [5].

\section{Stereotactic radiosurgery}

Has emerged as a new treatment option for the multidisciplinary management of metastases located within or adjacent to vertebral bodies and the spinal cord. The goals of stereotactic radiosurgery are to improve local control over conventional fractionated radiation therapy and to be effective for the treatment of previously irradiated lesions with an acceptable safety profile. Stereotactic radiosurgery offers several theoretical advantages as a treatment modality for spinal tumors: early treatment of these lesions before a patient becomes symptomatic and the stability of the spine, it avoids the need to irradiate large segments of the spinal cord, the early treatment of spinal lesions may obviate the need for extensive spinal surgery for decompression and fixation in these already debilitated patients and may also avoid the need to irradiate large segments of the spinal column, which is known to have a deleterious effect on bone marrow reserve in these patients. The avoidance of open surgery and the preservation of bone-marrow function facilitate continuous chemotherapy in this patient population. Other advantage is that treatment can be completed in a single day rather than over the course of several weeks. The limitations of stereotactic radiosurgery for spinal metastasis are: the quality of literature is poor; no randomized 


\section{Cancer Therapy \& Oncology International Journal}

controlled study has been conducted; stereotactic radiosurgery is more expensive than conventional RT $[61,62]$.

Assessing the response of bone metastases to therapy is difficult; the events in the healing process are slow to evolve and quite subtle, with sclerosis of lytic lesions only beginning to appear 3--6 months after the start of therapy and taking more than a year to mature. It is generally accepted that sclerosis of lytic metastases with no radiological evidence of new lesions constitutes tumor regression (a partial response). Confounding factors include the appearance of sclerosis in an area that was previously normal. After successful therapy for metastatic disease, the healing processes of new bone formation cause an initial increase in tracer uptake and scans carried out during this phase are likely to show increased production of new bone and isotope uptake gradually falls [33].

\section{Conclusion}

The presence of bone metastases is a sign of disseminated disease and foretells a short--term prognosis in cancer patients. The bone metastases have an important impact on patient's quality of life so, new strategies are necessary to prevent skeletal disease and palliate established skeletal events. A multidisciplinary approach should include medical oncologist, radiotherapist, pain control team, intervention radiologist, endocrinologist, orthopedic surgeon and psychologist. More studies are necessary to elucidate the interaction between tumor cell and bone microenvironment to reach new therapeutic interventions.

\section{References}

1. Chambers A, Naumov G, Varghese H, MacDonald IC, Groom AC, et al. (2001) Critical steps in hematogenous metastasis: an overview. Surg Oncol Clin N Am 10: 243-255.

2. Luzzi KJ, MacDonald IC, Schmidt EE, Chambers AF, Groom AC, et al. (1998) Multistep nature of metastatic inefficiency: dormancy of solitary cells after successful extravasation and limited survival of early micrometastases. Am J Pathol 153(3): 865-873.

3. Coleman R (2001) Metastatic bone disease: clinical features, pathophysiology and treatment strategies. Cancer Treat Rev 27(3) 165-176.

4. Cecchini M, Wetterwald A, Pluijm G, Thalmann G (2005) Molecular and Biological Mechanisms of Bone Metastasis. EAU Update Series 3: 214-226.

5. Selvaggi G, Scagliotti G (2005) Management of bone metastases in cancer: A review. Clinical Reviews in Oncology Hematology 56(3): 365-378.

6. Taube T, Elomaa I, Blomqvist C, Beneton MN, Kanis JA (1994) Histomorphometric evidence for osteoclast--mediated bone resorption in metastatic breast cancer. Bone 15(2):161-166.

7. Southby J, Kissin M, Danks J, Bennett RC, Martin TJ, et al. (1990) Immunohistochemical localization of parathyroid hormone--related protein in human breast cancer. Cancer Res 50(23): 7710-7716.

8. Kohno N, Kitazawa S, Fukase M, Ishikawa Y, Saitoh Y, et al. (1994) The expression of parathyroid hormone--related protein in human breast cancer with skeletal metastases. Surg Today 24(3): 215-220.
9. Dougall W, Glaccum M, Charrier K, Shen V, Bain S, et al. (1999) Rank is essential for osteoclast and lymph node development. Genes Dev 13(18): 2412-2424.

10. Keller E, Zhang J, Cooper C, Pienta KJ, Taichman RS, et al. (2001) Prostate carcinoma skeletal metastases: cross--talk between tumor and bone. Cancer Metastasis Rev 20: 333-349.

11. IwamuraM, Hellman J, Cockett A, Lilja H, Gershagen S (1996) Alteration of the hormonal bioactivity of parathyroid hormone-related protein (PTHrP) as a result of limited proteolysis by prostate--specific antigen. Urology 48(2): 317-325.

12. Yang $X$, Karsenty G (2002) Transcription factors in bone: developmental and pathological aspects. Trends Mol Med 8(7): 340 345 .

13. Coleman R, Seaman J (2001) The role of zoledronic acid in cancer: clinical studies in the treatment and prevention of bone metastasis. Semin Oncol 28: 11-16.

14. Berrettoni BA, Carter JR (1986) Mechanisms of cancer metastasis to bone. J Bone Joint Surg Am 68(2): 308-312.

15. Franks LM (1953) The spread of prostatic carcinoma to the bones. J Pathol Bacteriol 66: 91-93.

16. Springer TA (1994) Traffic signals for lymphocyte recirculation and leukocyte emigration: the multistep paradigm. Cell 76(2): 301-314.

17. Mazo IB, von Andrian UH (1999) Adhesion and homing of bloodborne cells in bone marrow microvessels. J Leukoc Biol 66: 25-32.

18. Sipkins DA, Wei X, Wu JW, Terry K Means, Andrew D, et al. (2005) In vivo imaging of specialized bone marrow endothelial microdomains for tumour engraftment. Nature 435: 969-973.

19. Balkwill F (2004) Cancer and the chemokine network. Nat Rev Cancer 4(7): 540-550.

20. Guo W, Giancotti FG (2004) Integrin signalling during tumour progression. Nat Rev Mol Cell Biol 5(10): 816-826.

21. Russel H, Hicks J, Okcu M, Nuchtern J (2004) CXCR4 expression in neuroblastoma primary tumors is associated with clinical presentation of bone and bone marrow metastases. J Pediatr Surg 39(10): 1506-1511.

22. Jones D, Nakashima T, Sanchez O, Wada T, Penninger JM, et al. (2006) Regulation of cancer cell migration and bone metastasis by RANKL. Nature 440: 692-696.

23. Papotti M, Kalebic T, Volante M, Borasio P, Scagliotti GV. et al. (2006) Bone sialoprotein is predictive of bone metastases in resectable non- small--cell lung cancer: a retrospective case--control study. J Clin Oncol 24: 4818-4824.

24. Paget S (1889) The distribution of secondary growths in cancer of the breast. Lancet 133(3421): 571-573.

25. Coleman R, Rubens R (1987) The clinical course of bone metastases from breast cancer. Br J Cancer 55: 61-66.

26. Coleman R, Smith P, Rubens R (1998) Clinical course and prognostic factors following recurrence from breast cancer. Br J Cancer 77(2): 336-340.

27. Robson M, Dawson N (1996) How is androgen dependent metastatic prostate cancer best treated? Haematol Oncol Clinics North Am 10(3): 727-747.

28. Maisano R, Pergolizzi S, Cascinu S (2001) Novel therapeutic approaches to cancer patients with bone metastasis. Crit Rev Oncol Hematol 40(3): 239-250.

29. Higinbotham N, Marcove R (1965) The management of pathological fractures. J Trauma 5(6): 792-799. 


\section{Cancer Therapy \& Oncology International Journal}

30. Nielson 0, Munro A, Tannock I (1991) Bone metastases: pathophysiology and management policy. J Clin Oncol 9(3): 509-524.

31. Hadji P, Aapro M, Body J, Guise T, Lipton A, et al. (2011) Management of aromatase inhibitor--associated bone loss in postmenopausal women with breast cancer: practical guidance for prevention and treatment. Ann Oncol 22(12): 2546-2555.

32. Clohisy D, Mantyh P (2003) Bone cancer pain. Clin Orthop Relat Res pp: S279-S288.

33. Coleman R, Body J, Aapro M, Hadji P, Herrstedt J, et al. (2014) Bone health in cancer patients: ESMO clinical practice guidelines. Ann Oncol 25: 124-137

34. Algra P, Bloem J, Tissing H, Arndt JW, Verboom LJ, et al. (1991) Detection of vertebral metastases: comparison between MRI and bone scintigraphy. Radiographics 11: 219-232.

35. Vinholes J, Coleman R, Eastell R (1996) Effects of bone metastases on bone metabolism: implications for diagnosis, imaging and assessment of response to cancer treatment. Cancer Treat Rev 22(4): 289-231.

36. Rosenthal D (1997) Radiologic diagnosis of bone metastases. Cancer 80: 1595-1607.

37. Evans A, Robertson J (2000) Magnetic resonance imaging versus radionuclide scintigraphy for screening in bone metastases. Clin Radiol 55(8): 653.

38. Marom E, McAdams H, Erasmus J, Coleman RE, Herndon JE, et al (1999) Staging non--small cell lung cancer with whole--body PET. Radiology 212(3): 803-809.

39. Yang S, Liang J, Lin F, et al. (2002) Comparing whole--body 18FDG PET and technetium--99m methylene diphosphonate bone scan to detect bone metastases in patients with breast cancer. J Cancer Res Clin Oncol 128: 325-328.

40. Schirrmeister H, Guhlmann A, Elsner K, Nüssle K, Reske SN, et al. (1999) Sensitivity in detecting osseous lesions depends on anatomic localization: planar bone scintigraphy versus 18F PET. J Nucl Med 40(10): 1623-1629.

41. Shreve P, Grossman H, Gross M, Wahl R (1996) Metastatic prostate cancer: initial findings of PET with 2--deoxy--2 (F--18) fluoro--d-glucose. Radiology 199(3): 751-755.

42. Rogers M, Xiong X, Ji X, Ebetino FH, Watts DJ, et al. (1997) Inhibition of growth of Dictyostelium discoideum amoebae by bisphosphonates is dependent on cellular uptake. Pharmacol Res 14(5): 625-630.

43. Rogers M, Watts D, Russel R (1997) Overview of bisphosphonates. Cancer 80: 1652-1660.

44. Body J, Coleman R, Piccart M (1996) Use of bisphosphonates in cancer patients. Cancer Treat Rev 22: 265-287.

45. Vinholes J, Purohit O, Abbey M, Eastell R, Coleman RE, et al. (1997) Relationships between biochemical and symptomatic response in double--blind trial of pamidronate for metastatic bone disease. Ann Oncol 8(12): 1243-1250.

46. Green JR, Rogers MJ (2002) Pharmacologic profile of zoledronic acid: a highly potent inhibitor of bone resorption. Drug Dev Res 55: 210224
47. Wellington K, Goa KL (2003) Zoledronic acid. A review of its use in the management of bone metastases and hypercalcemia of malignancy. Drugs 63(4): 417-437.

48. Steger G, Bartsch R (2011) Denosumab for the treatment of bone metastases in breast cancer: evidence and opinion. Ther Adv Med Oncol 3(5): 233-243.

49. Migliorati C, Epstein J, Abt E, Berenson J (2011) Osteonecrosis of the jaw and bisphosphonates in cancer: a narrative review. Nat Rev Endocrinol 7: 34-42.

50. Janjan N (1997) Radiation of bone metastases: conventional techniques and the role of systemic radiopharmaceuticals. Cancer 80: 1628-1645.

51. Tong C, Gillick L, Hendrickson F (1982) The palliation of symptomatic osseous metastases: final results of the study by the Radiation Therapy Oncology Group. Cancer 50(5): 893-899.

52. Salazar O, Rubin P, Hendrickson F, Mohiuddin M, Van Ess J, et al (1986) Single--dose half--body irradiation for palliation of multiple bone metastases from solid tumors: final Radiation Therapy Oncology Group report. Cancer 58: 29-36.

53. Poulter C, Cosmatos D, Rubin P, Weigensberg I, Rotman M, et al. (1992) A report of RTOG 8206: a phase III study of whether the addition of single dose hemibody irradiation to standard fractionated local field irradiation is more effective than local field irradiation alone in the treatment of symptomatic osseous metastases. Int J Radiat Oncol Biol Phys 23: 207-214.

54. Robinson R, Preston D, Baxter K (1993) Clinical experience with strontium 89 in prostatic and breast cancer patients. Semin Oncol 20 44-48.

55. Parker C, Nilsson S, Heinrich D, Logue M, Seke A, et al. (2013) Alpha emitter Radium--223 and survival in metastatic prostate cancer. N Engl J Med 18: 213-223.

56. American Cancer Society. Bone metastasis

57. Saliken J, McKinnon J, Gray R (1996) CT for monitoring cryotherapy. AJR Am JRoentgenol 166(4): 853-855.

58. Thacker P, Callstrom M, Curry T, Jayawant N Mandrekar, Thomas D Atwell, et al. (2011) Palliation of painful metastatic disease involving bone with image-- guided treatment: comparison of patients immediate response to radiofrequency ablation and cryoablation. AJR Am J Roentgenol 197: 510-515.

59. Callstrom M, Dupuy D, Solomon S, Paz-Fumagalli R, Hoffman C, et al. (2013) Percutaneous image--guided cryoablation of painful metastases involving bone: multicenter trial. Cancer 119(5): 10331041.

60. Lee R, Saylor P, Michaelson M, S Michael Rothenberg, Malgorzata E Smas, et al. (2013) A dose--ranging study of cabozantinib in men with castration--resistant prostate cancer and bone metastases. Clin. Cancer Res 19: 3088-3094.

61. Jhaveri P, Teh B, Bloch C, Butler EB, Paulino AC, et al. (2008) Stereotactic body radiotherapy in the management of painful bone metastases. Oncology 22(7): 782-788.

62. Sohn S, Chung C (2012) The Role of Stereotactic Radiosurgery in Metastasis to the Spine. J Korean Neurosurg Soc 51(1): 1-7. 
This work is licensed under Creative Commons Attribution 4.0 License DOI: $10.19080 /$ CTOIJ.2017.04.555637
Your next submission with Juniper Publishers will reach you the below assets

- Quality Editorial service

- Swift Peer Review

- Reprints availability

- E-prints Service

- Manuscript Podcast for convenient understanding

- Global attainment for your research

- Manuscript accessibility in different formats

( Pdf, E-pub, Full Text, Audio)

- Unceasing customer service

Track the below URL for one-step submission https://juniperpublishers.com/online-submission.php 